\title{
ALGUNAS ESTRATEGIAS PARA EXPRESAR CORTESÍA EN LOS ESCRITOS DE PETICIÓN EN EL ESPAÑOL PERUANO Y COLOMBIANO ${ }^{1}$
}

\author{
Masamichi Kawaguchi \\ Universidad de Estudios Extranjeros de Kobe (Japón) \\ m-kawaguchi@inst.kobe-cufs.ac.jp
}

Recibido: 14/02/2021 - Aprobado: 06/06/2021- Publicado: 30/07/2021

DOI: doi.org/10.17533/udea.lyl.n80a17

\begin{abstract}
Resumen: En este artículo se describen y comparan las estrategias de hablantes nativos de español peruanos y colombianos utilizadas para expresar cortesía en textos de petición escritos. Asimismo, con el fin de determinar tendencias de uso, el trabajo adapta (i) la teoría de la cortesía y (ii) la metodología de la pragmática interlingüística al análisis de la variedad lingüística. La exploración de los mensajes redactados por los participantes para cuatro situaciones ficticias reveló la existencia de similitudes y diferencias asociadas con la fuerza relativa entre el emisor y el receptor, y con el grado de imposición.
\end{abstract}

Palabras clave: cortesía; variación lingüística; pragmática; comunicación escrita; enseñanza del español.

\section{SOME STRATEGIES TO EXPRESS POLITENESS IN WRITTEN REQUESTS IN PERUVIAN AND COLOMBIAN SPANISH}

\begin{abstract}
In this paper, the strategies used by Peruvian and Colombian native speakers of Spanish to express politeness in written requests are described and compared. Also, in order to determine usage trends, it applies (i) politeness theory and (ii) interlanguage pragmatic methodology to the analysis of linguistic variety. The exploration of the messages produced by the participants for four fictitious situations revealed similarities and differences associated with both the relative power dynamics between sender and receiver, and the degree of imposition.
\end{abstract}

Key words: politeness; linguistic variation; pragmatics; written communication; teaching of Spanish.

1. La presente investigación ha sido financiada por JSPS KAKENHI (Subvención para investigaciones científicas por el Ministerio de Educación, Cultura, Deportes, Ciencias y Tecnologías de Japón. Número de investigación: 19K13296). Asimismo, este artículo está basado en la ponencia presentada en la $433^{\text {a }}$ Reunión del Círculo de Lingüística Hispánica de Kansai, celebrada de forma virtual el 19 de diciembre de 2020. Queremos expresar nuestros sinceros agradecimientos a los participantes de dicha reunión por sus valiosos consejos y comentarios. 


\section{Introducción}

$\mathrm{E}$ ste trabajo aborda las estrategias de hablantes nativos de español peruanos y colombianos utilizadas para expresar cortesía en textos de petición escritos. El objetivo es describir las tendencias observables en los mensajes redactados por dichos hablantes - en el contexto de cuatro situaciones ficticias-y definir características de composición.

La estructura de este artículo es la siguiente: en el primer apartado discutiremos brevemente nuestro marco teórico y una selección de estudios sobre la variación intercultural de la cortesía. Posteriormente, en el segundo apartado, examinaremos la relación entre la cortesía y la comunicación escrita, mientras que en el tercer apartado describiremos la metodología de nuestro análisis. Finalmente, el cuarto apartado presentará el análisis de los datos.

Por otra parte, este artículo forma parte de un proyecto destinado a desarrollar un método para enseñar composición a hablantes japoneses estudiantes de español, el cual incluirá diferencias pragmáticas entre las lenguas.

\section{Marco teórico: la cortesía y su variación intercultural}

En este apartado presentaremos la teoría de la cortesía de Brown y Levinson (1987), que constituirá la base teórica de nuestro análisis. Seguidamente, discutiremos algunos estudios sobre la variación intercultural de la cortesía.

\subsection{La teoría de la cortesía de Brown y Levinson}

Se considera que la cortesía sirve para mantener una buena relación social entre los interlocutores a la hora de comunicarse: por ejemplo, cuando un hablante solicita algo a su oyente, es posible que este se sienta ofendido si lo hace de manera brusca, pero si lo hace cortés o adecuadamente, no ocurre eso.

Se han propuesto algunas teorías con el fin de esclarecer los principios que regulan la cortesía. Entre ellas, la más conocida y desarrollada es la planteada por Penélope Brown y Stephen Levinson. El concepto más relevante de su teoría es el de imagen social (en inglés, face). Según Escandell (2013), esta idea se relaciona con la noción de que «[c]ada individuo tiene y reclama para sí una cierta imagen pública (un cierto prestigio) que quiere conservar» (Escandell, 2013, p. 154).

Este constructo presenta dos subcategorías: imagen negativa e imagen positiva. Escandell (2013) las describió de la siguiente manera: «I) negativa: deseo de tener libertad de acción, de no sufrir imposiciones por parte de los demás, de dominar el propio territorio»; y «II) positiva: deseo de ser apreciado por los demás, y de que 
Algunas estrategias para expresar cortesía en los escritos de petición en el español peruano y colombiano otros compartan los mismos deseos» ( p. 155). En otras palabras, la imagen negativa constituye el deseo de los hablantes de que no se les impida hacer lo que quieren, y la imagen positiva es el de ser percibido como una persona amable y generosa.

Esta teoría sugiere que las interacciones sociales conllevan un cierto riesgo de amenaza a la imagen social de aquellos quienes se relacionan (en inglés, face risk). Dicho riesgo es el resultado de la suma de la distancia social ( $\mathrm{D}[$ distance $])$ entre los interlocutores, la fuerza relativa $(\mathrm{P}$, por el término en inglés [power]) entre dichos interlocutores y el grado de imposición ${ }^{2}$ del acto en la cultura en la que tiene lugar la comunicación $(\mathrm{R}$, por el término en inglés [ranking of imposition]). Aquí detallaremos brevemente el segundo, la fuerza relativa (P), y el tercero, el grado de imposición del acto en la cultura en la que tiene lugar la comunicación, (R), ya que estas dos variables guardan estrecha relación con nuestra investigación que se presentará en el apartado 3. La fuerza relativa, (P), se refiere a la posición social en la que se encuentra el oyente con respecto al hablante: por ejemplo, para un empleado es complicado rechazar algo que le solicita su superior. En cuanto al grado de imposición del acto en la cultura en la que tiene lugar la comunicación, (R), es mayor cuando el acto se considera, en una determinada cultura, más costoso o difícil en algún sentido. Por ejemplo, se supone que, en muchas culturas, la R será mayor al pedir prestado a alguien un carro que un bolígrafo, porque un carro es de precio más elevado, hay un riesgo de accidente, etc. Según esta noción, el tipo de relación entre los hablantes conllevará que una misma enunciación de petición (como al pedirse prestado un bolígrafo) implique diferentes niveles de riesgo de amenaza a la imagen. Por eso, es común prestar más atención a la forma empleada cuando se solicita algo a un desconocido, puesto que la distancia social (D) es mayor que cuando el interlocutor es un amigo.

En realidad, existen numerosas críticas a este modelo de Brown y Levinson, muchas de las cuales ponen una objeción a su universalidad. Una de las propuestas realizadas en el mundo hispanohablante con el fin de desarrollar la teoría de Brown y Levinson es la de la Dra. Diana Bravo, quien insiste en la necesidad de tomar en consideración los contextos socioculturales y plantea los conceptos de autonomía y de afiliación (Bravo, 1999; 2003; 2008). Sus definiciones al respecto son las siguientes: «1. autonomía: abarca todos aquellos comportamientos que están relacionados con cómo una persona desea verse y ser vista por los demás como un individuo con contorno propio dentro del grupo. (Bravo, 2008, p. 579)», «2. afiliación: agrupa aquellos comportamientos mediante los cuales una persona manifiesta cómo desea verse y ser vista por los demás en cuanto a aquellas características que identifican con el grupo (p. 579)». Según esta autora, estas son unas categorías «vacías» que se rellenan en cada contexto sociocultural donde tiene lugar la comunicación (Bravo, 2008, p. 578). ${ }^{3}$ Esta óptica nos parece de vital importancia para dar cuenta de la variación de la cortesía en distintas culturas. Aun así, en esta investigación, optaremos por la teoría más básica de Brown y Levinson, especialmente con respecto al diseño de la investigación, ya que, a nuestro juicio, aunque justamente sea este aspecto el que se critica, el planteamiento de Brown y Levinson es más concreto y, por tanto, el fruto obtenido de este marco nos parece más provechoso en el ámbito de la enseñanza del

\footnotetext{
2. Término extraído de Ballesteros Martín (1999).

3. Sobre otras críticas y el desarrollo de la teoría de Brown y Levinson, véase Jang (2011).
} 
Algunas estrategias para expresar cortesía en los escritos de petición en el español peruano y colombiano español como lengua extranjera, que es la meta final de nuestro proyecto, tal y como comentaremos más adelante.

En el siguiente subapartado, discutiremos algunas investigaciones en las que se trata la variación intercultural de las expresiones de cortesía.

\subsection{Variación intercultural de las expresiones de la cortesía}

Numerosos autores han analizado la variación de la expresión de la cortesía en distintas culturas lingüísticas en base a la teoría de Brown y Levinson y a una metodología derivada de ella. Haverkate (2003, p. 60), al respecto, comentó: «[...] nuestro foco de interés será el estudio de la dimensión intercultural del proceso comunicativo, o sea, en términos más específicos, el análisis de la cortesía». En esta línea, el autor comparó las expresiones de cortesía de españoles y holandeses. Veamos los ejemplos citados en este trabajo:

(1)

AM - Que ya no puedes ser patinadora o exploradora, ¿no?

$\mathrm{GN}-\mathrm{O}$ asi parece entonces.

AM - O así parece, claro. (Nichols, 1989, p. 113, como se citó en Haverkate, 2003).

(2)

Bautista: - Me dijeron que la tos había desaparecido y que de fiebre sólo tiene cuarenta y cinco grados.

Dalmacio — ¡Pues síque está mejor! (Beinhauer, 1985, como se citó en Haverkate, 2003) (las cursivas son nuestras)

En el diálogo en (1) el hablante AM repite lo que GN ha enunciado. Según Haverkate, al repetir las palabras del interlocutor, el hablante enfatiza que este concuerda con el punto de vista de aquel. Esta es una estrategia empleada comúnmente en España, pero no en Países Bajos.

En el ejemplo (2), el autor indica que la palabra pues — al comienzo del enunciado en cursiva— funciona como marca de ironía, por lo que la frase completa recibe dicha interpretación. En neerlandés, por otro lado, no suelen utilizarse marcas de este tipo, lo que es característico de una cultura en la que usualmente se reserva una distancia entre los interlocutores. A partir de este y otros datos lingüísticos, Haverkate concluyó que la cultura española da importancia a la cortesía positiva, mientras que la neerlandesa prioriza la negativa.

Otro estudio que siguió la misma línea de investigación fue el de Curcó (1998), el cual comparó los dialectos mexicano y español. En dicho trabajo, la autora aclaró que los mexicanos parecen ser más sensibles al riesgo de amenaza a la imagen positiva del interlocutor que los españoles. Esta característica los llevaría a preferir enunciaciones que cuentan con estrategias para salvaguardar dicha imagen, es decir, estrategias para mantener una imagen positiva (en inglés, positive politeness strategy). Según la autora, un mayor número de mexicanos consideraría la oración en (3) como inadecuada desde el punto de vista de la cortesía, puesto que se emplea una forma imperativa para realizar una petición: ${ }^{4}$

4. Se consideraría que la oración condicional que sigue al imperativo se agrega con el propósito de no amenazar la imagen negativa del oyente. 
Algunas estrategias para expresar cortesía en los escritos de petición en el español peruano y colombiano

(3)

Mire, aquí hay varios errores. Repitalo, si no le importa (la directora de una empresa a su secretaria)(Curcó, 1998, p. 148).

En este apartado, hemos resumido los aspectos más relevantes de la teoría de la cortesía de Brown y Levinson —nuestro marco teórico- y, seguidamente, hemos explorado una selección de estudios sobre la variación interlingüística de la cortesía. En el siguiente apartado, abordaremos la relación entre la cortesía y el otro eje de nuestra investigación: la comunicación escrita.

\section{La comunicación escrita y la cortesía}

Nuestro análisis se enfoca en las estrategias usadas por hablantes nativos de español peruanos y colombianos para expresar cortesía al producir enunciados escritos. Este apartado constará de dos secciones: primero, clarificaremos las razones por las que hemos seleccionado la comunicación escrita como objeto de investigación y especificaremos las diferencias que se hallan entre la comunicación escrita y la oral; y segundo, discutiremos algunos de los estudios publicados sobre este tema en los últimos años.

\subsection{La comunicación escrita}

Como ya hemos mencionado, la mayor parte de los numerosos trabajos sobre las diferencias regionales de la cortesía se enfocan en la comunicación oral. No obstante, como se discutió en Kawaguchi (2016, 2018), la sociedad actual se comunica mediante correos electrónicos y mensajes enviados desde aplicaciones móviles, tanto en el contexto laboral como privado: hoy nos comunicamos escribiendo, cuando hace unas décadas lo hacíamos con llamadas telefónicas. Montolío (2014), en su guía de redacción Manual de escritura académica y profesional, comentó lo siguiente sobre esta situación:

Desde entonces [nota del autor: el año 2000] hasta hoy, el fenómeno de la comunicación escrita no ha hecho más que ampliarse: escribimos más que nunca, en más ámbitos profesionales, a cualquier hora del día, individualmente y también de manera colectiva, en todo tipo de soportes tecnológicos, con propósitos diversos, para destinatarios muy variados (personales y profesionales; individuales y masivos). En el siglo xxI la escritura muestra más que nunca su enorme complejidad cognitiva, antropológica, tecnológica y de elaboración (Montolío, 2014, p. 8).

Esta autora ya había publicado las obras Manual de escritura académica y Manual práctico de escritura académica, en 1999 y 2000, respectivamente. Sin embargo, es evidente que el cambio social mencionado en esta sección fue tomado en consideración en la edición del año 2014 (citada anteriormente).

Como ya señalamos, numerosas investigaciones se han llevado a cabo sobre el tema de la cortesía en el contexto de la expresión oral, y claramente existe la posibilidad de que sus conclusiones sean aplicables al contexto escrito. Sin embargo, Montolío (2014) también ha afirmado que «Escribir para un lector de pantallas [...] requiere activar 
Algunas estrategias para expresar cortesía en los escritos de petición en el español peruano y colombiano unas estrategias de escritura nuevas» (p. 7). Asimismo, Yui (2005) comentó lo siguiente con respecto al uso de correos electrónicos, un medio típicamente usado para la comunicación escrita:

A excepción de aquellas situaciones en las que se intercambian oraciones simples — como en los mensajes de texto-, enviar un correo electrónico conlleva el envío simultáneo de una cantidad de discurso considerable. En dichas circunstancias, surge la necesidad de tomar medidas para prevenir el rechazo del receptor o interlocutor, mantener la relación, y al mismo tiempo, lograr que este no se lleve la impresión de que el hablante es egoísta o desagradable. De cierta forma, el uso del correo electrónico requiere un mayor cuidado al utilizar expresiones de cortesía que el requerido al «hablar» cara a cara (Yui, 2005, p. 194). (La traducción es nuestra).

En este contexto, consideramos imprescindible llevar a cabo una investigación enfocada específicamente en el uso de las expresiones de cortesía en la comunicación escrita. Los resultados de este análisis serán incorporados en la elaboración de un método para la enseñanza de composición española a extranjeros, lo cual constituye la meta principal de nuestro proyecto.

Acerca del concepto de la teoría de la cortesía de Brown y Levinson y los tres componentes del riesgo de la amenaza a la imagen social -D, P y R-descritos en el subapartado 2.1., los consideramos aplicables también a la comunicación escrita. Pues, hay un emisor y un receptor en una interacción, ya sea oral o escrita, entre los cuales debe existir necesariamente una distancia social (D) y diferencia de la fuerza relativa (P). Y si se trata de un acto que amenaza la imagen, es obvio que hay un cierto grado de imposición en cada cultura.

\subsection{Estudios previos sobre la cortesía en la comunicación escrita}

Como mencionamos en el subapartado anterior, existen ciertas diferencias fundamentales entre la comunicación oral y la escrita, y una de ellas es el uso de la cortesía. Esto ha llevado a la reciente publicación de diversos trabajos sobre las estrategias de cortesía utilizadas en la comunicación escrita de hablantes nativos de distintas variedades del español. Algunos de estos trabajos serán discutidos en este subapartado.

En primer lugar, César Vera (2014) realizó una investigación centrada en la comunicación por correo electrónico entre 16 universitarios venezolanos y sus profesores. La autora sugirió que el género de los hablantes, su distancia social y el nivel de formalidad del mensaje son aspectos que influyen en el uso de expresiones atenuantes relacionadas con la atenuación.

Por otra parte, es importante mencionar el trabajo sobre variación geográfica llevado a cabo por Cantamutto y Vela Delfa (2019). En este artículo, las autoras analizaron correos electrónicos redactados por universitarios españoles y argentinos, dirigidos a una variedad de receptores (profesores, oficinistas, amigos y compañeros). El trabajo concluyó, entre otras cosas, que los universitarios argentinos utilizan un mayor número de expresiones corteses que los españoles, excepto en los mensajes dirigidos a profesores; que los españoles emplean la expresión gracias de antemano, en contextos en los que los argentinos usan desde ya gracias; y que los estudiantes de ambos países frecuentemente utilizan la frase (te/le) escribo.

El presente estudio es semejante al de Cantamutto y Vela Delfa (2019) en tanto ambos se enfocan en la variación 
Algunas estrategias para expresar cortesía en los escritos de petición en el español peruano y colombiano geográfica. No obstante, el presente análisis es singular, puesto que forma parte de un proyecto exhaustivo que, por un lado, proveerá una descripción comprensiva de las tendencias de uso de la cortesía en regiones importantes del mundo hispanohablante y, por otro, aplicará estos resultados a la enseñanza de español como lengua extranjera.

En los siguientes apartados, presentaremos la metodología de nuestra investigación y el análisis de los datos.

\section{Metodología}

Este estudio se realizó entre finales de agosto y principios de septiembre del año 2019, gracias a la participación de dos grupos de adultos hablantes nativos de español: 31 peruanos residentes en las ciudades de Lima y Cuzco (13 hombres y 18 mujeres), y 22 colombianos residentes en Medellín (9 hombres y 13 mujeres). ${ }^{56}$ El método utilizado fue el de Pruebas de Finalización del Discurso (en inglés, Discourse Completion Test o DCT). En esta tarea, los participantes debieron redactar cuatro mensajes escritos, ${ }^{7}$ cada uno asociado a una situación ficticia. Optamos por esta metodología porque tiene la ventaja de que nos permite controlar las condiciones en cada situación, es decir, las dos dimensiones que nos interesan en esta investigación: la fuerza relativa y el grado de imposición del acto en la cultura en la que tiene lugar la comunicación. Otra posibilidad sería el empleo de un corpus de datos reales de mensajes escritos, pero en ese caso nos resultaría difícil saber las condiciones y relaciones humanas entre los interlocutores en las situaciones comunicativas en las que se intercambian los mensajes.

Preparamos un formulario en línea en el que los participantes escribieron los mensajes basados en las condiciones que mostraremos más adelante. La manera concreta en la que recabamos los datos es la siguiente: los limeños se reunieron en una sala e introdujeron los mensajes con una computadora, mientras que los residentes de las otras dos ciudades (Cuzco y Medellín) lo hicieron con una computadora o con un celular de forma telemática. ${ }^{8}$ Se acordó con los participantes que dichos mensajes podrían ser citados en futuras publicaciones bajo condiciones de anonimidad.

Todas las situaciones ficticias incluidas solicitaban al participante producir una petición, la cual constituye uno de los actos de habla más abordados en los estudios sobre la cortesía. Esto último se debe a que las peticiones implican un riesgo relativamente alto de amenaza a la imagen del interlocutor y, por tanto, frecuentemente suponen el uso de estrategias atenuantes vinculadas al cuidado de la imagen del destinatario. Las situaciones ficticias incluidas en el estudio son las siguientes:

5. Los grupos etarios de los participantes son los siguientes: Perú: 20-29 años (17 personas), 30-30 años (12 personas), 40-49 años (2 personas); Colombia: 20-29 años (13 personas), 30-30 años (8 personas), 40-49 años ( 1 persona).

6. En esta investigación no se han tenido en cuenta la edad y el sexo de los participantes. Este punto lo dejaremos para futuros estudios.

7. No especificamos si se trata de un correo electrónico o un mensaje de texto en un celular, puesto que esos medios varían dependiendo de a quién se dirige el mensaje.

8. Todos los participantes fueron seleccionados con la ayuda de nuestros colaboradores residentes en cada ciudad. 
$<$ Situación 1>

Usted tiene que hacer la mudanza dentro de un mes y necesita que algunas personas lo/la ayuden ese día. Por favor, escriba un mensaje de texto o un correo electrónico para pedírselo A UN AMIGO SUYO o A UNA AMIGA SUYA.

$<$ Situación 2>

Usted tiene que hacer la mudanza dentro de un mes y necesita que algunas personas lo/la ayuden ese día. Usted les ha preguntado a varios amigos suyos, pero todos estarán ocupados ese día y la única posibilidad que le queda es pedírselo a su jefe/jefa. Por favor, escriba un mensaje de texto o un correo electrónico para pedírselo A SU JEFE/JEFA (Piense que no le queda más remedio que pedírselo a su jefe/jefa).

$<$ Situación $3>$

Usted va a organizar una pequeña fiesta en su casa, pero no está seguro/ra de qué comida y bebida hay que comprar y le gustaría que alguien lo/la acompañara al supermercado para hacer la compra juntos. Por favor, escriba un mensaje de texto o un correo electrónico para pedírselo A UN AMIGO SUYO O A UNA AMIGA SUYA.

$<$ Situación 4>

Usted va a organizar una fiesta en su casa, pero no está seguro/ra de qué comida y bebida hay que comprar y le gustaría que alguien lo/la acompañara al supermercado para hacer la compra juntos. Usted les ha preguntado a varios amigos suyos, pero todos estarán ocupados y la única posibilidad que le queda es pedírselo a su jefe/jefa. Por favor, escriba un mensaje de texto o un correo electrónico para pedírselo A SU JEFE/JEFA (Piense que no le queda más remedio que pedírselo a su jefe/jefa).

La tarea incluida en este estudio fue preparada dentro del marco teórico de Brown y Levinson (1987). Como ya mencionamos, según los autores, el riesgo de amenaza a la imagen social es el resultado de la suma de la distancia social entre los interlocutores (D [distance]), la fuerza relativa (P [power]) entre los interlocutores y el grado de imposición del acto en la cultura en la que tiene lugar la comunicación ( $\mathrm{R}$ [ranking of imposition]). En las situaciones presentadas en nuestro estudio existen dos destinatarios: amigo/amiga o jefe/jefa. Si bien es posible que el emisor no perciba diferencias de fuerza relativa (P) con un amigo/amiga, ante un jefe/jefa sí es usualmente considerado como de una mayor fuerza relativa. Por otra parte, colaborar con una mudanza es una actividad que requiere una inversión de tiempo y energía considerables, por lo que conlleva un grado de imposición mayor que la de simplemente acompañar a alguien al mercado. De esta manera, aunque todas las situaciones requieren producir peticiones, la tarea controla el factor de riesgo de amenaza a la imagen. Las características de la tarea pueden ser resumidas en el Esquema 1:

\begin{tabular}{|l|c|c|}
\hline & Fuerza relativa & Grado de imposición del acto \\
\hline Situación 1 & menor & mayor \\
\hline Situación 2 & mayor & mayor \\
\hline Situación 3 & menor & menor \\
\hline Situación $\mathbf{4}$ & mayor & menor \\
\hline
\end{tabular}

Esquema 1. Relación entre el nivel de exigencia de las situaciones planteadas y el grado de imposición de los actos demandados 
A continuación, en la Tabla 1 se presenta el número de respuestas válidas producidas por los participantes de cada país y para cada tarea:

\begin{tabular}{|c|c|c|c|c|}
\hline Situación & Tarea & Destinatario & Perú & Colombia \\
\hline $\mathbf{1}$ & Mudanza & Amigo/Amiga & 28 & 20 \\
\hline $\mathbf{2}$ & Mudanza & Jefe/Jefa & 28 & 21 \\
\hline $\mathbf{3}$ & Compra & Amigo/Amiga & 30 & 22 \\
\hline $\mathbf{4}$ & Compra & Jefe/Jefa & 30 & 21 \\
\hline
\end{tabular}

Tabla 1. Relación del número de respuestas válidas producidas por los participantes de cada país y para cada tarea

Para definir la metodología de nuestro análisis, consultamos los trabajos de Blum-Kulka et al. (1989) y Trosborg (1995), y adaptamos sus procesos para el estudio de la comunicación oral al estudio de la comunicación escrita. El trabajo de Blum-Kulka et al. (1989) dividió el enunciado de petición en tres partes: i) acto nuclear ${ }^{9}$ (en inglés, head act), ii) alertador ${ }^{10}$ (en inglés, alerter) y iii) movimiento de apoyo ${ }^{11}$ (en inglés, supportive move). El acto nuclear constituye la sección de la oración en la que se describe el contenido de la petición. El alertador, por otro lado, es aquella sección en la que se llama la atención del interlocutor por medio de un vocativo, lo que no será incluido en este trabajo, puesto que estudiaremos únicamente mensajes escritos. Por último, el movimiento de apoyo es el conjunto de fragmentos de la oración que bien refuerza o atenúa la petición comprendida en el acto nuclear.

En nuestro análisis, el primer paso consistió en distinguir los actos nucleares y los movimientos de apoyo en todos los mensajes redactados por nuestros participantes. En el ejemplo en (4), la oración en cursiva constituiría el acto nuclear, mientras que todo el resto correspondería al movimiento de apoyo. Posteriormente, los mensajes fueron seccionados según las nociones de fórmulas semánticas (en inglés, semantic formulae, [Blum-Kulka, 1989]), y cada sección fue etiquetada. Ahora bien, como detallaremos en el siguiente apartado, en este trabajo solo han sido etiquetadas las fórmulas semánticas utilizadas frecuentemente en los enunciados de petición, que son las que aparecen en la Tabla 2:

(4)

Hola (nombre de la destinataria), espero que estés muy bien [saludo]. Te escribo para hacerte una pregunta... [preparador] resulta que el próximo mes debo mudarme [explicación de la consecuencia] y, como fue tan repentino, no he podido encontrar quien me ayude [razón]. Quisiera saber si tu esposo (nombre del esposo de la destinataria) y tu (sic) podrían venir a echarme una mano [acto nuclear, deseo [quisiera saber]]. La mudanza sería el domingo

9. Término extraído de Ballesteros Martín (1999).

10. La traducción de este término es nuestra.

11. Término extraído de Ballesteros Martín (1999). 
Algunas estrategias para expresar cortesía en los escritos de petición en el español peruano y colombiano 14, ese día la cena corre por mi cuenta así que no hay de que (sic) preocuparse [mención de la remuneración]. ¡Espero poder contar con ustedes! Quedo pendiente de tu respuesta. [esperanza asociada a la respuesta] Gracias. [agradecimiento] (Colombia, 17). ${ }^{12}$

En el próximo apartado, presentaremos los datos obtenidos y el análisis.

\section{Datos y análisis}

\subsection{Acto nuclear}

Para comenzar, en este subapartado se presentarán los datos relacionados con la sección de los mensajes que contiene el acto nuclear. El análisis se centrará especialmente en el uso de aquellas fórmulas semánticas que, según estudios anteriores (Kawaguchi, 2016; 2018), aparecen con frecuencia en los escritos que expresan petición. Además, es importante aclarar lo siguiente: si bien la metodología de Blum-Kulka et al. y Trosborg fue propuesta originalmente para el análisis de temas de pragmática interlingüística, es decir, con el objetivo de contrastar los fenómenos pragmáticos de distintas lenguas, nuestro objeto de investigación es el español peruano y el español colombiano. En otras palabras, este estudio se enfoca en el estudio de la variación pragmática en el mismo idioma, aunque en variedades diferentes. Además, puesto que uno de los propósitos de esta investigación es aplicar sus resultados a la enseñanza de ELE, recurrimos al uso de expresiones lingüísticas concretas para la subclasificación y el etiquetado de ciertas fórmulas semánticas.

Las Tablas 2 y 3 resumen el número de participantes en cada país que emplearon cada fórmula semántica en el acto nuclear, y los porcentajes con respecto a la totalidad de los mensajes producidos.

\begin{tabular}{|c|c|c|c|c|c|c|c|c|c|}
\hline \multirow[b]{2}{*}{ Fórmula semántica } & \multirow[b]{2}{*}{ Formas } & \multicolumn{2}{|c|}{ Perú 1 (28) } & \multicolumn{2}{|c|}{ Perú 2 (28) } & \multicolumn{2}{|c|}{ Perú 3 (30) } & \multicolumn{2}{|c|}{ Perú 4 (30) } \\
\hline & & $\%$ & núm. & $\%$ & núm. & $\%$ & núm. & $\%$ & núm. \\
\hline Imperativo & ---- & 0.0 & 0 & 3.6 & 1 & 20.0 & 6 & 0.0 & 0 \\
\hline \multirow[t]{3}{*}{ Verbo performativo de petición } & Pedir & 0.0 & 0 & 7.1 & 2 & 0.0 & 0 & 6.7 & 2 \\
\hline & Solicitar & 0.0 & 0 & 0.0 & 0 & 0.0 & 0 & 3.3 & 1 \\
\hline & Rogar & 0.0 & 0 & 0.0 & 0 & 3.3 & 1 & 0.0 & 0 \\
\hline \multirow{3}{*}{$\begin{array}{c}\text { Necesidad + Verbo performativo } \\
\text { de petición }\end{array}$} & Necesito pedir & 0.0 & 0 & 0.0 & 0 & 0.0 & 0 & 0.0 & 0 \\
\hline & $\begin{array}{c}\text { Tengo la necesidad de } \\
\text { pedir }\end{array}$ & 0.0 & 0 & 0.0 & 0 & 0.0 & 0 & 0.0 & 0 \\
\hline & $\begin{array}{l}\text { Verse en la necesidad } \\
\text { de solicitar }\end{array}$ & 0.0 & 0 & 3.6 & 1 & 0.0 & 0 & 3.3 & 1 \\
\hline
\end{tabular}

12. La información que aparece entre paréntesis incluye (i) la nacionalidad del participante y (ii) el código de identificación asignado. 
Algunas estrategias para expresar cortesía en los escritos de petición en el español peruano y colombiano

\begin{tabular}{|c|c|c|c|c|c|c|c|c|c|}
\hline \multirow{5}{*}{$\begin{array}{l}\text { Deseo }+ \text { Verbo performativo de } \\
\text { petición }\end{array}$} & Quiero pedir & 0.0 & 0 & 0.0 & 0 & 0.0 & 0 & 0.0 & 0 \\
\hline & Quería pedir & 3.6 & 1 & 10.7 & 3 & 3.3 & 1 & 0.0 & 0 \\
\hline & Quisiera pedir & 0.0 & 0 & 0.0 & 0 & 0.0 & 0 & 0.0 & 0 \\
\hline & Deseo pedir & 0.0 & 0 & 3.6 & 1 & 0.0 & 0 & 0.0 & 0 \\
\hline & Me gustaría pedir & 0.0 & 0 & 7.1 & 2 & 0.0 & 0 & 3.3 & 1 \\
\hline \multirow{3}{*}{$\begin{array}{c}\text { Posibilidad + Verbo performativo } \\
\text { de petición }\end{array}$} & Puedo pedir & 0.0 & 0 & 0.0 & 0 & 0.0 & 0 & 0.0 & 0 \\
\hline & Podía pedir & 0.0 & 0 & 0.0 & 0 & 0.0 & 0 & 0.0 & 0 \\
\hline & Podría pedir & 0.0 & 0 & 0.0 & 0 & 0.0 & 0 & 0.0 & 0 \\
\hline Necesidad & Necesito & 14.3 & 4 & 3.6 & 1 & 3.3 & 1 & 3.3 & 1 \\
\hline \multirow[t]{7}{*}{ Deseo } & Quiero que & 0.0 & 0 & 0.0 & 0 & 0.0 & 0 & 0.0 & 0 \\
\hline & Quisiera que & 0.0 & 0 & 0.0 & 0 & 0.0 & 0 & 0.0 & 0 \\
\hline & Quería que & 0.0 & 0 & 0.0 & 0 & 3.3 & 1 & 3.3 & 1 \\
\hline & Quiero saber & 0.0 & 0 & 0.0 & 0 & 0.0 & 0 & 0.0 & 0 \\
\hline & Quería saber & 7.1 & 2 & 0.0 & 0 & 0.0 & 0 & 0.0 & 0 \\
\hline & Quisiera saber & 0.0 & 0 & 0.0 & 0 & 0.0 & 0 & 3.3 & 1 \\
\hline & Me gustaría que... & 3.6 & 1 & 0.0 & 0 & 0.0 & 0 & 10.0 & 3 \\
\hline \multirow{10}{*}{$\begin{array}{l}\text { Pregunta sobre posibilidad o ca- } \\
\text { pacidad }\end{array}$} & ¿Puedes...? & 3.6 & 1 & 0.0 & 0 & 10.0 & 3 & 0.0 & 0 \\
\hline & ¿Podrías...? & 17.9 & 5 & 7.1 & 2 & 16.7 & 5 & 6.7 & 2 \\
\hline & $\begin{array}{c}\text { ¿Crees que }+ \\
\text { [posibilidad]...? }\end{array}$ & 7.1 & 2 & 10.7 & 3 & 3.3 & 1 & 10.0 & 3 \\
\hline & $\begin{array}{c}\text { Preguntar }+ \\
\text { [posibilidad] }\end{array}$ & 0.0 & 0 & 7.1 & 2 & 0.0 & 0 & 3.3 & 1 \\
\hline & ¿No podrías...? & 0.0 & 0 & 0.0 & 0 & 0.0 & 0 & 0.0 & 0 \\
\hline & ¿Será que puedes? & 0.0 & 0 & 0.0 & 0 & 0.0 & 0 & 0.0 & 0 \\
\hline & ¿Será que podrías? & 0.0 & 0 & 0.0 & 0 & 0.0 & 0 & 0.0 & 0 \\
\hline & ¿Es posible...? & 0.0 & 0 & 0.0 & 0 & 0.0 & 0 & 0.0 & 0 \\
\hline & ¿Sería posible? & 0.0 & 0 & 0.0 & 0 & 0.0 & 0 & 0.0 & 0 \\
\hline & ¿Podría (yo)...? & 0.0 & 0 & 0.0 & 0 & 0.0 & 0 & 3.3 & 1 \\
\hline \multirow[t]{6}{*}{ Pregunta sobre la intención } & ¿Vas a...? & 0.0 & 0 & 0.0 & 0 & 0.0 & 0 & 0.0 & 0 \\
\hline & ¿Haces...? & 0.0 & 0 & 0.0 & 0 & 3.3 & 1 & 0.0 & 0 \\
\hline & ¿Será que haces...? & 0.0 & 0 & 0.0 & 0 & 0.0 & 0 & 0.0 & 0 \\
\hline & ¿Será que hacemos? & 0.0 & 0 & 0.0 & 0 & 0.0 & 0 & 0.0 & 0 \\
\hline & ¿Quieres...? & 0.0 & 0 & 0.0 & 0 & 0.0 & 0 & 0.0 & 0 \\
\hline & ¿Harías...? & 0.0 & 0 & 0.0 & 0 & 3.3 & 1 & 0.0 & 0 \\
\hline \multirow[t]{2}{*}{ Pregunta sobre tiempo } & ¿Tienes tiempo...? & 10.7 & 3 & 0.0 & 0 & 6.7 & 2 & 3.3 & 1 \\
\hline & $\begin{array}{c}\text { ¿Será que tienes } \\
\text { tiempo...? }\end{array}$ & 0.0 & 0 & 0.0 & 0 & 0.0 & 0 & 0.0 & 0 \\
\hline \multirow[t]{2}{*}{ Expresión de agradecimiento } & Te agradecería... & 3.6 & 1 & 7.1 & 2 & 0.0 & 0 & 6.7 & 2 \\
\hline & Sería de gran ayuda... & 0.0 & 0 & 0.0 & 0 & 0.0 & 0 & 3.3 & 1 \\
\hline
\end{tabular}


Algunas estrategias para expresar cortesía en los escritos de petición en el español peruano y colombiano

\begin{tabular}{|c|c|c|c|c|c|c|c|c|c|}
\hline Invitación & ---- & 0.0 & 0 & 0.0 & 0 & 6.7 & 2 & 6.7 & 2 \\
\hline Uso de «Por favor» & ---- & 14.3 & 4 & 14.3 & 4 & 26.7 & 8 & 16.7 & 5 \\
\hline
\end{tabular}

Tabla 2. Resultados del acto nuclear obtenidos por los particpantes de Perú) ${ }^{13}$

\begin{tabular}{|c|c|c|c|c|c|c|c|c|c|}
\hline \multirow[b]{2}{*}{ Fórmula semántica } & \multirow[b]{2}{*}{ Formas } & \multicolumn{2}{|c|}{$\begin{array}{c}\text { Colombia } 1 \\
(20)\end{array}$} & \multicolumn{2}{|c|}{$\begin{array}{c}\text { Colombia } 2 \\
(21)\end{array}$} & \multicolumn{2}{|c|}{$\begin{array}{c}\text { Colombia } 3 \\
(22)\end{array}$} & \multicolumn{2}{|c|}{$\begin{array}{c}\text { Colombia } 4 \\
\text { (21) }\end{array}$} \\
\hline & & $\%$ & núm. & $\%$ & núm. & $\%$ & núm. & $\%$ & núm. \\
\hline Imperativo & ----- & 0.0 & 0 & 0.0 & 0 & 4.5 & 1 & 0.0 & 0 \\
\hline \multirow[t]{3}{*}{ Verbo performativo de petición } & Pedir & 0.0 & 0 & 19.0 & 4 & 0.0 & 0 & 4.8 & 1 \\
\hline & Solicitar & 0.0 & 0 & 14.3 & 3 & 0.0 & 0 & 0.0 & 0 \\
\hline & Rogar & 0.0 & 0 & 0.0 & 0 & 0.0 & 0 & 0.0 & 0 \\
\hline \multirow{3}{*}{$\begin{array}{l}\text { Necesidad }+ \text { Verbo performativo de pe- } \\
\text { tición }\end{array}$} & Necesito pedir & 0.0 & 0 & 0.0 & 0 & 0.0 & 0 & 0.0 & 0 \\
\hline & Tengo la necesidad de pedir & 0.0 & 0 & 4.8 & 1 & 0.0 & 0 & 0.0 & 0 \\
\hline & $\begin{array}{l}\text { Verse en la necesidad de solic- } \\
\text { itar }\end{array}$ & 0.0 & 0 & 0.0 & 0 & 0.0 & 0 & 0.0 & 0 \\
\hline \multirow[t]{5}{*}{ Deseo + Verbo performativo de petición } & Quiero pedir & 0.0 & 0 & 0.0 & 0 & 0.0 & 0 & 0.0 & 0 \\
\hline & Quería pedir & 5.0 & 1 & 4.8 & 1 & 9.1 & 2 & 9.5 & 2 \\
\hline & Quisiera pedir & 5.0 & 1 & 4.8 & 1 & 0.0 & 0 & 4.8 & 1 \\
\hline & Deseo pedir & 0.0 & 0 & 0.0 & 0 & 0.0 & 0 & 0.0 & 0 \\
\hline & Me gustaría pedir & 0.0 & 0 & 0.0 & 0 & 0.0 & 0 & 0.0 & 0 \\
\hline \multirow{3}{*}{$\begin{array}{l}\text { Posibilidad }+ \text { Verbo performativo de pe- } \\
\text { tición }\end{array}$} & Puedo pedir & 0.0 & 0 & 0.0 & 0 & 0.0 & 0 & 0.0 & 0 \\
\hline & Podía pedir & 0.0 & 0 & 0.0 & 0 & 0.0 & 0 & 0.0 & 0 \\
\hline & Podría pedir & 0.0 & 0 & 0.0 & 0 & 0.0 & 0 & 4.8 & 1 \\
\hline Necesidad & Necesito & 10.0 & 2 & 0.0 & 0 & 9.1 & 2 & 0.0 & 0 \\
\hline \multirow[t]{7}{*}{ Deseo } & Quiero que & 0.0 & 0 & 0.0 & 0 & 0.0 & 0 & 0.0 & 0 \\
\hline & Quisiera que & 0.0 & 0 & 0.0 & 0 & 0.0 & 0 & 4.8 & 1 \\
\hline & Quería que & 0.0 & 0 & 0.0 & 0 & 0.0 & 0 & 0.0 & 0 \\
\hline & Quiero saber & 0.0 & 0 & 0.0 & 0 & 0.0 & 0 & 0.0 & 0 \\
\hline & Quería saber & 0.0 & 0 & 0.0 & 0 & 0.0 & 0 & 4.8 & 1 \\
\hline & Quisiera saber & 10.0 & 2 & 4.8 & 1 & 0.0 & 0 & 14.3 & 3 \\
\hline & Me gustaría que... & 0.0 & 0 & 0.0 & 0 & 0.0 & 0 & 0.0 & 0 \\
\hline
\end{tabular}

13. En las Tablas 2 y 3, en la sección de la fórmula semántica deseo + verbo performativo, no hemos hecho distinción entre los diferentes verbos performativos (por ejemplo, pedir, solicitar, rogar, etc.), y todos han sido representados con el verbo pedir. De la misma manera, en la sección de pregunta sobre la intención, todos los verbos principales son expresados con el verbo hacer. 
Algunas estrategias para expresar cortesía en los escritos de petición en el español peruano y colombiano

\begin{tabular}{|c|c|c|c|c|c|c|c|c|c|}
\hline \multirow[t]{10}{*}{ Pregunta sobre posibilidad o capacidad } & ¿Puedes...? & 0.0 & 0 & 4.8 & 1 & 0.0 & 0 & 4.8 & 1 \\
\hline & ¿Podrías...? & 15.0 & 3 & 9.5 & 2 & 9.1 & 2 & 9.5 & 2 \\
\hline & ¿Crees que [posibilidad]...? & 0.0 & 0 & 0.0 & 0 & 0.0 & 0 & 0.0 & 0 \\
\hline & Preguntar+[posibilidad] & 0.0 & 0 & 9.5 & 2 & 0.0 & 0 & 0.0 & 0 \\
\hline & ¿No podrías...? & 5.0 & 1 & 0.0 & 0 & 0.0 & 0 & 0.0 & 0 \\
\hline & ¿Será que puedes? & 15.0 & 3 & 0.0 & 0 & 9.1 & 2 & 0.0 & 0 \\
\hline & ¿Será que podrías? & 5.0 & 1 & 0.0 & 0 & 0.0 & 0 & 0.0 & 0 \\
\hline & ¿Es posible...? & 0.0 & 0 & 0.0 & 0 & 0.0 & 0 & 4.8 & 1 \\
\hline & ¿Sería posible? & 0.0 & 0 & 0.0 & 0 & 0.0 & 0 & 9.5 & 2 \\
\hline & ¿Podría (yo)...? & 0.0 & 0 & 0.0 & 0 & 0.0 & 0 & 0.0 & 0 \\
\hline \multirow[t]{6}{*}{ Pregunta sobre la intención } & ¿Vas a...? & 10.0 & 2 & 0.0 & 0 & 13.6 & 3 & 0.0 & 0 \\
\hline & ¿Haces...? & 0.0 & 0 & 0.0 & 0 & 4.5 & 1 & 0.0 & 0 \\
\hline & ¿Será que haces...? & 5.0 & 1 & 0.0 & 0 & 4.5 & 1 & 0.0 & 0 \\
\hline & ¿Será que hacemos? & 0.0 & 0 & 0.0 & 0 & 9.1 & 2 & 0.0 & 0 \\
\hline & ¿Quieres...? & 0.0 & 0 & 0.0 & 0 & 9.1 & 2 & 0.0 & 0 \\
\hline & ¿Harías...? & 0.0 & 0 & 0.0 & 0 & 0.0 & 0 & 0.0 & 0 \\
\hline \multirow[t]{2}{*}{ Pregunta sobre tiempo } & ¿Tienes tiempo...? & 5.0 & 1 & 0.0 & 0 & 4.5 & 1 & 0.0 & 0 \\
\hline & ¿Será que tienes tiempo...? & 0.0 & 0 & 4.8 & 1 & 0.0 & 0 & 4.8 & 1 \\
\hline \multirow[t]{2}{*}{ Expresión de agradecimiento } & Te agradecería... & 0.0 & 0 & 9.5 & 2 & 0.0 & 0 & 0.0 & 0 \\
\hline & Sería de gran ayuda... & 5.0 & 1 & 0.0 & 0 & 0.0 & 0 & 0.0 & 0 \\
\hline Invitación & ----- & 5.0 & 1 & 0.0 & 0 & 9.1 & 2 & 4.8 & 1 \\
\hline Uso de «Por favor» & $\begin{array}{ll}---- \\
\end{array}$ & 10.0 & 2 & 9.5 & 2 & 9.1 & 2 & 14.3 & 3 \\
\hline
\end{tabular}

Tabla 3. Resultados del acto nuclear obtenidos por los participantes de Colombia

A continuación, incluiremos algunos ejemplos del uso de cada fórmula semántica detectada a nivel de acto nuclear en nuestro análisis: ${ }^{14}$

Imperativo

(5) [...] acompáñame al supermercado para comprar todo lo que me falta (Perú, 5).

(6) Ayúdame en (sic) elegir las cosas que les agrada (Perú, 31).

Verbo performativo de petición

(7) [...] le pido el favor si me puede acompañar a comprar las cosas (Colombia, 3).

(8) [...] le pido encarecidamente me brinde su apoyo (Perú, 10).

Deseo + verbo performativo de petición

(9) [...] le deseo solicitar su ayuda con su movilidad y poder trasladar todo (Perú, 12).

(10) [...] quería pedirte que me acompañes a comprar la comida y las bebidas para tener todo listo(Colombia, 10).

Deseo

(11) [...] quisiera que me acompañaras a realizar las compras para la reunión de esta semana (Colombia, 7).

(12) [...] me gustaría que usted pudiera ayudarme con las compras (Perú, 10).

14. Las cursivas son nuestras. 
Algunas estrategias para expresar cortesía en los escritos de petición en el español peruano y colombiano

Pregunta sobre posibilidad o capacidad

(13) [...] podrías acompañarme mas (sic) temprano a comprar la comida y los tragos? (Perú, 8).

(14) ¿Me podrías ayudar con unas cosas de la mudanza por fa? (Colombia, 1).

Pregunta sobre la intención

(15) [...] me va a echar una manito? (Colombia, 2).

(16) ¿Me acompañarías al supermercado esta noche? (Perú, 29).

Con respecto a los resultados, deseamos discutir varios puntos. En primer lugar, la fórmula semántica pregunta sobre posibilidad o capacidad surgió de manera relativamente frecuente en el contexto del acto nuclear, tanto entre participantes peruanos como colombianos, independientemente de la fuerza relativa $(P)$ o el grado de imposición de la petición en cuestión $(R)$. Esta fórmula fue usada principalmente en la situación 1 (pedir ayuda a un amigo/ una amiga con una mudanza) con la forma condicional (por ejemplo, ¿Podría(s)...?) por los participantes de ambos países (17,9 \% en Perú y 15,0 \% en Colombia), por lo cual, en este aspecto, no se observa una gran diferencia entre los hablantes de ambos países.

(17) [...] me podría ayudar con la mudanza que tengo planeada para el próximo mes? (Colombia, 7).

(18) [...] podrías venir un poco antes, a eso de las 5 y ayudarme a comprar lo que serviremos en la fiesta, por favor? (Perú, 21).

En segundo lugar, la fórmula semántica deseo aparece mayoritariamente en la estructura quisiera saber..., en la forma imperfecto del subjuntivo de cortesía, y especialmente entre los participantes colombianos. Otra expresión de deseo notable es me gustaría que..., que se usa especialmente en la situación 4 (pedir a su jefe/jefa que le acompañe a hacer la compra) entre los participantes peruanos. ${ }^{15}$

A continuación, discutiremos las expresiones consideradas como altamente directivas (término derivado de la palabra en inglés directness) (Trosborg, 1995), que son definidas como aquellas en las que el acto nuclear contiene una expresión que es claramente interpretable como una petición. Dicho de otra manera, las expresiones en imperativo y las que incluyen verbos como pedir, solicitar, etc. exhiben un alto grado de directividad. Ahora bien, lo que más nos llama la atención es la alta frecuencia del uso de la fórmula semántica imperativo —la opción más directa - en la situación 3 (pedir a un amigo/una amiga que le acompañe a hacer la compra) entre los participantes peruanos (20\%), y el mínimo uso de dicha fórmula entre los colombianos (utilizada una vez $-4,5 \%$ del total— y solo en la situación 3). Por ejemplo:

15. En el grupo de fórmulas semánticas relacionadas con la de deseo, la estructura querer que... apareció de manera poco frecuente, y quiero que..., en presente del modo indicativo, no fue utilizada en lo absoluto. Este punto es relevante desde el punto de vista de ELE, que es, tal y como mencionamos anteriormente, la meta general de nuestro proyecto, puesto que en el trabajo de Kawaguchi (2018), prácticamente la mitad de los participantes (estudiantes japoneses de español como lengua extranjera) emplearon esta fórmula a la hora de realizar una petición. Si bien la investigación de Kawaguchi (2018) incluyó situaciones en las que el contenido de la petición era diferente de aquellas en el presente trabajo, nuestra experiencia como docentes de español nos lleva a suponer que los estudiantes japoneses emplearían dicha frase con igual frecuencia. Nuestro proyecto planea llevar a cabo una investigación similar con este tipo de estudiante. 
Algunas estrategias para expresar cortesía en los escritos de petición en el español peruano y colombiano

(19) Ayúdame en (sic) elegir las cosas que les agrada (Perú, 31).

(20) [...] acompañame (sic) a comprar comida y bebidas para la fiesta que sera (sic) en mi casa [...] (Perú, 14).

En la situación 3, los participantes colombianos prefirieron usar la fórmula preguntas sobre la intención con estructuras como ¿vas $a \ldots$ ? (13,6\%), ¿será que hacemos...? $(9,1 \%)$, etc.

(21) ¿Me va a acompañar por fa y me ayuda a elegir? (Colombia, 1).

(22) [...] para la reunión de esta semana, será que me acompañas al supermercado para comprar las cosas [...]

(Colombia, 7).

De esto se deduce que, en el contexto de peticiones que no representan una gran imposición para una persona sin fuerza relativa, los hablantes peruanos aceptan el uso de expresiones más directas que aquellas usadas por los hablantes colombianos. Dicho de otra manera, desde el punto de vista de la cortesía, podemos juzgar que los colombianos tienden a evitar la amenaza a la imagen negativa del interlocutor en comparación con los peruanos en la situación en cuestión.

Otro aspecto relevante relacionado con las expresiones directas es que, en la situación 2, el 33,3\% de los participantes colombianos emplearon la fórmula semántica verbo performativo de petición (el 19 \% utilizó el verbo pedir y el 14,3\%, el verbo solicitar).

(23) [...] no me ha quedado más que perdirle por favor que usted me ayude en este proceso [...] (Colombia, 13).

Por otro lado, si bien el 7,1 \% de los participantes peruanos también optaron por el uso de esta fórmula en dicha situación (con el verbo pedir), los datos señalan que el 21,4 \% utilizó este tipo de verbo en combinación con una estructura de deseo como quería pedir, deseo pedir y me gustaría pedir (10,7 \%, 3,6 \% y 7,1\%, respectivamente):

(24) Es por eso que quería pedirle si usted fuera tan amable de poder ayudarme [...] (Perú, 11).

(25) Es por ello que me gustaría pedir su ayuda para realizar este traslado (Perú, 23).

Atendiendo a estos datos, los hablantes de los dos países optan por emplear los verbos performativos de petición en una situación donde el interlocutor tiene una mayor fuerza relativa respecto al emisor; sin embargo, se nota la diferencia de que más colombianos los emplean independientemente, mientras que los peruanos prefieren aplicarlo combinado con una expresión de deseo. De momento, no es posible valorar este fenómeno relacionándolo con la teoría de la cortesía, por lo que nos limitamos a indicar la tendencia.

Con respecto a las expresiones empleadas por solo un grupo de participantes, nos gustaría enfocarnos en ¿será que...?, entre los participantes colombianos, y crees que + posibilidad, ${ }^{16}$ entre los peruanos. Por un lado, la forma ¿será que...? apareció únicamente en aquellos mensajes dirigidos a un amigo o amiga, mientras que crees que + posibilidad surgió en todas las situaciones. Esto sugiere que ¿será que...? constituye una estructura de atenuación 16. Si bien es posible emplear crees que + posibilidad en Colombia, esta estructura no surgió en los mensajes redactados por los participantes de dicho país en esta investigación. 
Algunas estrategias para expresar cortesía en los escritos de petición en el español peruano y colombiano que es utilizada en los mensajes dirigidos a personas sin una fuerza relativa mayor a la del hablante:

(26) ¿Será que me podés ayudar? (Colombia, 18).

(27) Usted cree que podría ir conmigo al supermercado a comprarla? (Perú, 2).

Además de las incidencias de las diversas fórmulas semánticas, también cuantificamos el uso de la expresión por favor o porfa, un elemento prototípico indiscutible en las expresiones de petición. Los resultados indican que, aunque es una expresión poco frecuente entre los hablantes de ambos países, su uso parece ser un poco más común entre los hablantes peruanos. Esto se observa de manera especial en la situación 3 (26,7 \% entre los participantes peruanos y $9,1 \%$ entre los colombianos).

(28) ¿puedes acompañarme a hacer las compras por favor? (Perú, 24).

(29) $(=(14))$ ¿Me podrías ayudar con unas cosas de la mudanza por fa? (Colombia, 1).

En este subapartado, hemos analizado y discutido las características del acto nuclear de una selección de mensajes ficticios redactados por hablantes de español peruanos y colombianos. En la siguiente sección, examinaremos los movimientos de apoyo.

\subsection{El movimiento de apoyo}

Como ya mencionamos, en este subapartado se incluyen los resultados obtenidos en relación con los movimientos de apoyo. Con el fin de clasificar este elemento, en lugar de enfocarnos en formas lingüísticas concretas, solamente nos centraremos en las fórmulas semánticas. Asimismo, consultando las obras de BlumKulka et al. (1989) y Trosborg (1995), analizaremos aquellas fórmulas semánticas que aparecen de manera más frecuente en los mensajes de petición según Kawaguchi $(2016$; 2018). El resultado está sintetizado en las Tablas 4 y 5 .

\begin{tabular}{|c|c|c|c|c|c|c|c|c|}
\hline & \multicolumn{2}{|c|}{ Perú 1 (28) } & \multicolumn{2}{c|}{ Perú 2 (28) } & \multicolumn{2}{c|}{ Perú 3 (30) } & \multicolumn{2}{c|}{ Perú 4 (30) } \\
\hline & \% & núm. & \% & núm. & \% & núm. & \% & núm. \\
\hline Saludo & 78.6 & 22 & 82.1 & 23 & 66.7 & 20 & 70.0 & 21 \\
\hline Preparador & 39.3 & 11 & 42.9 & 12 & 26.7 & 8 & 30.0 & 9 \\
\hline Explicación de la circunstancia & 89.3 & 25 & 96.4 & 27 & 56.7 & 17 & 76.7 & 23 \\
\hline Razón & 53.6 & 15 & 53.6 & 15 & 73.3 & 22 & 80.0 & 24 \\
\hline Mención de la remuneración & 42.9 & 12 & 21.4 & 6 & 10.0 & 3 & 6.7 & 2 \\
\hline Minimización de la imposición & 7.1 & 2 & 7.1 & 2 & 0.0 & 0 & 3.3 & 1 \\
\hline Impedimento al rechazo & 0.0 & 0 & 10.7 & 3 & 0.0 & 0 & 6.7 & 2 \\
\hline Agradecimiento & 35.7 & 10 & 42.9 & 12 & 10.0 & 3 & 36.7 & 11 \\
\hline
\end{tabular}


Algunas estrategias para expresar cortesía en los escritos de petición en el español peruano y colombiano

\begin{tabular}{|c|c|c|c|c|c|c|c|c|}
\hline Petición de disculpas & 3.6 & 1 & 28.6 & 8 & 0.0 & 0 & 26.7 & 8 \\
\hline Temas ajenos a la petición & 0.0 & 0 & 0.0 & 0 & 3.3 & 1 & 3.3 & 1 \\
\hline $\begin{array}{c}\text { Esperanza asociada a la } \\
\text { respuesta }\end{array}$ & 50.0 & 14 & 39.3 & 11 & 46.7 & 14 & 43.3 & 13 \\
\hline $\begin{array}{c}\text { Expresión de pena } \\
\text { Expresión de vergüenza }\end{array}$ & 0.0 & 0 & 3.6 & 1 & 0.0 & 0 & 0.0 & 0 \\
\hline Uso de «Por favor» & 32.1 & 9 & 10.7 & 3 & 30.0 & 9 & 13.3 & 4 \\
\hline
\end{tabular}

Tabla 4. Resultados de los movimientos de apoyo obtenidos por los participantes de Perú

\begin{tabular}{|c|c|c|c|c|c|c|c|c|}
\hline & \multicolumn{2}{|c|}{ Colombia 1 (20) } & \multicolumn{2}{c|}{ Colombia 2 (21) } & \multicolumn{2}{c|}{ Colombia 3 (22) } & \multicolumn{2}{c|}{ Colombia 4 (21) } \\
\hline & $\mathbf{\%}$ & $\mathbf{n u ́ m}$ & $\mathbf{\%}$ & $\mathbf{n u ́ m . ~}$ & $\mathbf{\%}$ & núm. & \% & núm. \\
\hline Saludo & 85.0 & 17 & 90.5 & 19 & 72.7 & 16 & 95.2 & 20 \\
\hline Preparador & 40.0 & 8 & 33.3 & 7 & 22.7 & 5 & 28.6 & 6 \\
\hline Explicación de la circunstancia & 80.0 & 16 & 85.7 & 18 & 63.6 & 14 & 85.7 & 18 \\
\hline Razón & 60.0 & 12 & 23.8 & 5 & 81.8 & 18 & 66.7 & 14 \\
\hline Mención de la remuneración & 50.0 & 10 & 14.3 & 3 & 9.1 & 2 & 0.0 & 0 \\
\hline Minimización de la imposición & 15.0 & 3 & 33.3 & 7 & 0.0 & 0 & 33.3 & 7 \\
\hline Impedimento al rechazo & 0.0 & 0 & 19.0 & 4 & 0.0 & 0 & 0.0 & 0 \\
\hline Agradecimiento & 50.0 & 10 & 76.2 & 16 & 27.3 & 6 & 52.4 & 11 \\
\hline Petición de disculpas & 0.0 & 0 & 33.3 & 7 & 0.0 & 0 & 23.8 & 5 \\
\hline Temas ajenos a la petición & 0.0 & 0 & 4.8 & 1 & 0.0 & 0 & 14.3 & 3 \\
\hline Esperanza asociada a la respuesta & 45.0 & 9 & 47.6 & 10 & 9.1 & 2 & 33.3 & 7 \\
\hline Expresión de pena & 0.0 & 0 & 9.5 & 2 & 0.0 & 0 & 4.8 & 1 \\
\hline Expresión de vergüenza & 0.0 & 0 & 23.8 & 5 & 0.0 & 0 & 4.8 & 1 \\
\hline Uso de «Por favor» & 25.0 & 5 & 0.0 & 0 & 13.6 & 3 & 0.0 & 0 \\
\hline
\end{tabular}

Tabla 5. Resultados de los movimientos de apoyo obtenidos por los participantes de Colombia

Antes de comenzar con el análisis de los datos, deseamos clarificar el significado de dos fórmulas semánticas cuyas denominaciones pueden causar confusión: minimización de la imposición (en inglés, imposition minimizer) e impedimento al rechazo (en inglés, disarmer ${ }^{17}$ ), propuestas por Blum-Kulka et al. (1989). Según estos autores, la fórmula semántica minimización de la imposición se define de la siguiente manera: «The speaker tries to reduce the imposition placed on the hearer by his request.» (Blum-Kulka et al., 1989, p. 288). Uno de los ejemplos

17. Las traducciones al español de las denominaciones de las fórmulas semánticas acuñadas por Blum-Kulka (1989) son nuestras. Ahora bien, la palabra disarmer ha sido traducida en este estudio como impedimento al rechazo en base a la traducción al japonés propuesta por Shimizu (2009). Consideramos que esta versión del término en cuestión ofrece mayor claridad que aquella ofrecida por una traducción literal. 
Algunas estrategias para expresar cortesía en los escritos de petición en el español peruano y colombiano incluidos en el trabajo de Blum-Kulka et al. es el siguiente:

(30) Would you give me a lift but only if you are going my way (Blum-Kulka et al., 1989, p. 288).

En la oración en (30), el hablante solicita al interlocutor que lo lleve en su auto. Sin embargo, el hablante agrega una condición que, de no cumplirse, exime al interlocutor de aceptar la petición. Por otro lado, para los autores antes mencionados, la fórmula semántica impedimento al rechazo se entiende como lo siguiente: «The speaker tries to remove any potential objections the hearer might raise upon being confronted with the request.» (Blum-Kulka et al., 1989, p. 287). Por ejemplo:

(31) I know you don't like lending out your notes, but could you make an exception this time? (Blum-Kulka et al., 1989, p. 287).

En (31), el hablante solicita al interlocutor que le preste sus apuntes, especificando con anticipación que él/ ella sabe que al oyente no le gusta hacerlo. En otras palabras, la petición en cuestión es expresada a pesar de las sabidas preferencias del interlocutor, lo cual obstaculiza su rechazo.

Analicemos ahora el resultado resumido en las Tablas 4 y 5, específicamente las fórmulas semánticas, cuyo uso es destacable. Con respecto a las similitudes entre los dos países, en primer lugar, la fórmula semántica preparador, usada para sondear la disponibilidad del receptor a aceptar la petición, tiende a emplearse levemente más en las situaciones 1 y 2 (pedir ayuda para una mudanza) que en las 3 y 4 (pedir a una persona que le acompañe a hacer la compra) entre los hablantes de ambos países. Esto sugiere que dicha fórmula suele usarse más frecuentemente en los mensajes de petición que implican una mayor imposición al interlocutor con el objetivo de salvaguardar la imagen negativa del interlocutor. Las frases (32) y (33) ejemplifican el uso de esta fórmula al inicio del mensaje:

(32) Oye, te puedo pedir un favor? (Colombia, 8).

(33) La presente es para solicitarle un favor de carácter personal (Perú, 4).

Por otro lado, la fórmula mención de la remuneración fue incluida de manera significativa en los mensajes correspondientes a la situación 1, tanto entre los hablantes peruanos como en los colombianos. Específicamente, esta fórmula fue seleccionada para la situación 1 por el 42,9 \% de los hablantes peruanos, y por el 50,0 \% de los hablantes colombianos.

(34) Después salimos y yo te invito ;) (Colombia, 5).

(35) Prometo invitarte un almuerzo y unas cervezas luego (Perú, 19).

Es posible concluir que los hablantes de ambos países usan esta fórmula al producir mensajes de petición que implican una alta imposición a un interlocutor sin mayor fuerza relativa. Sin embargo, también es posible colegir lo siguiente: en esta investigación, la petición que conlleva menor imposición al interlocutor es aquella relacionada con acompañar al hablante a la compra para una fiesta. En este caso, la expectativa es que, luego de la 
Algunas estrategias para expresar cortesía en los escritos de petición en el español peruano y colombiano compra, el interlocutor sea bienvenido a participar en la fiesta, lo que implica que no sería necesario ofrecer una recompensa adicional. Puede ser por eso por lo que pocos participantes incluyeron este detalle en sus mensajes de petición del acompañamiento a la compra.

La fórmula petición de disculpas aparece en los mensajes dirigidos a un jefe o jefa entre los hablantes de ambos países. Es posible suponer que ambos grupos de hablantes suelen disculparse a la hora de solicitar algo a una persona con mayor fuerza relativa, sin importar el grado de la imposición. Veamos el siguiente ejemplo:

(36) Disculpe las molestias (Colombia, 22).

(37) lo (sic) siento mucho si lo incomodo con mi petición (Perú, 24).

Desde el punto de vista de la cortesía, pedir disculpas es un acto que implica el riesgo de amenazar la imagen positiva del mismo emisor, puesto que reconoce la molestia al interlocutor causada por su petición, pero, aun así, se observa que es una estrategia de la mitigación común entre hablantes de ambos países.

Ahora vamos a analizar las diferencias. La fórmula minimización de la imposición es empleada con mayor frecuencia entre los hablantes colombianos y en los mensajes dirigidos a un jefe o jefa. Consideramos que es lógico tratar de evitar que una petición dirigida a una persona con mayor fuerza relativa represente una imposición para esta. Según nuestros datos, dicha tendencia es más visible entre los hablantes colombianos que entre los peruanos.

(38) [...] si le es posible y no le resulta incómodo ni inconveniente (Colombia, 13).

(39) Si no tiene tiempo no hay problema, yo lo resuelvo sola (Colombia, 8).

A continuación, discutiremos el uso de la fórmula semántica agradecimiento. Dar las gracias utilizando expresiones como gracias de antemano es usual al producir mensajes de petición. En nuestro estudio, el uso de estas expresiones fue más frecuente entre los hablantes colombianos que entre los peruanos.

(40) De antemano muchas gracias (Perú, 15).

(41) Mil gracias (Colombia, 12).

Por último, la fórmula expresión de la vergüenza es usada mayormente por los hablantes colombianos, y por solo el 3,3\% de los peruanos (únicamente un caso). Sería posible que esta fórmula estuviera relacionada con la fórmula expresión de la pena ${ }^{18}$ ya que según el Diccionario de la lengua española (23 ${ }^{\text {a }}$ edición en línea), la palabra pena también significa vergüenza en Colombia y algunas zonas americanas. A pesar de esto, esta última fórmula tampoco es frecuente entre los hablantes peruanos y claras diferencias se advierten entre ambos grupos de hablantes. Todos los casos registrados en los mensajes producidos por los hablantes colombianos son dirigidos a un jefe o una jefa, lo que sugiere que estos hablantes emplean dicha fórmula en peticiones dirigidas a personas

18. Aun así, distinguimos la expresión de la pena y la de la vergüenza en nuestra clasificación porque no tenemos la evidencia de que estas dos fórmulas sean empleadas en el mismo sentido. 
Algunas estrategias para expresar cortesía en los escritos de petición en el español peruano y colombiano con mayor fuerza relativa. A pesar de que, en términos de la cortesía, el reconocimiento de la vergüenza puede consistir en una amenaza a la imagen positiva del propio hablante, un $23,8 \%$ de los participantes colombianos eligieron esta fórmula.

(42) Me avergüenza pedirle el favor (Colombia, 4).

(43) Es vergonzoso para mi (sic) hacer esta petición (Colombia, 17).

Similarmente al análisis del acto nuclear, en esta sección del estudio también se enumeraron las incidencias de las expresiones por favor o porfa. Estas expresiones tienden a emplearse en los mensajes dirigidos a amigos —un resultado un tanto inesperado - $\mathrm{y}$, en total, parecen ser utilizadas más frecuentemente por los hablantes peruanos que por los colombianos. Lo interesante es que en el movimiento de apoyo, igual que en el acto nuclear, más peruanos tienden a utilizar esta expresión que los colombianos. Un punto que diferencia el uso de esta expresión en el movimiento de apoyo de los que se observan en el acto nuclear es que en aquel, a diferencia de en este, el porcentaje en la situación 1 es tan alto como en la situación 3 (32,1 \% y 30,0 \%, respectivamente).

En esta sección hemos analizado los resultados obtenidos con respecto a los movimientos de apoyo y hemos discutido algunos aspectos notables. Entre las fórmulas semánticas mencionadas en este subapartado, si bien varias son utilizadas de manera similar por los hablantes de ambos países, sí se aprecian algunas diferencias. Por ejemplo, la fórmula minimización de la imposición es de uso más frecuente entre los hablantes colombianos, particularmente en los mensajes dirigidos a un jefe o jefa. Al emplear esta fórmula, el emisor intenta evitar que el receptor se sienta obligado a acceder a la petición, es decir, dicha fórmula es considerada como una estrategia típica para evitar amenazar su imagen negativa. Otra fórmula de uso frecuente entre los hablantes colombianos es agradecimiento, que contribuye a reforzar la imagen positiva del interlocutor. De todo esto podemos concluir que, según los datos que disponemos, los hablantes colombianos prestan mayor atención a proteger la imagen del receptor.

\section{Conclusiones}

En este trabajo, hemos descrito y comparado mensajes de petición redactados por hablantes nativos de español de Perú y Colombia. Las tendencias que hemos identificado son las siguientes:

\subsection{Acto nuclear}

- Tanto los hablantes peruanos como los colombianos usan principalmente la fórmula semántica pregunta sobre posibilidad o capacidad, especialmente en la forma condicional (por ejemplo, ¿Podrías...?). Es decir, no se aprecia una gran diferencia en este aspecto desde el punto de vista de la cortesía sino que se trata de una similitud.

- En caso de utilizarse la fórmula semántica deseo, usualmente se seleccionan las estructuras quisiera 
Algunas estrategias para expresar cortesía en los escritos de petición en el español peruano y colombiano saber..., especialmente entre los hablantes colombianos, y me gustaría que, entre los peruanos, en una situación con una fuerza relativa mayor del interlocutor y un grado de imposición menor.

- En ausencia de diferencias de fuerza relativa entre los interlocutores, y en el contexto de una petición que no implique una gran imposición para el receptor, los hablantes peruanos utilizan la forma imperativa con mayor frecuencia que los colombianos, lo cual nos permite notar la consciencia mayor por parte de los colombianos de no amenazar la imagen negativa del interlocutor respecto a por los peruanos.

- Los hablantes colombianos tienden a seleccionar verbos performativos directos (por ejemplo, pedir y solicitar) al expresar una petición de gran imposición dirigida a una persona con mayor fuerza relativa. En la misma situación, los hablantes peruanos emplean verbos similares pero combinados con una expresión de deseo.

- La estructura ¿será que...? es utilizada solo por hablantes colombianos en mensajes dirigidos a personas sin mayor fuerza relativa que el emisor. Por otro lado, los hablantes peruanos tienden a usar la estructura ¿crees que + posibilidad? en todos los contextos provistos en nuestro análisis.

- El uso de por favor o porfa es más frecuente entre los hablantes peruanos. Ahora bien, esta diferencia se vuelve particularmente notable al expresar una petición de baja imposición a una persona de menor o igual fuerza relativa.

\subsection{Movimiento de apoyo}

- Tanto los hablantes peruanos como los colombianos tienden a emplear la fórmula preparador ligeramente más a la hora de realizar una petición que supone más imposición.

- Es posible deducir que en ambos países, se prefiere ofrecer una remuneración para una petición que entraña una imposición alta a una persona que no tiene una fuerza relativa mayor. Pero, a causa de la particularidad de la situación ficticia presentada en esta investigación, es difícil asegurarlo.

- Se advierte la tendencia a aplicar la fórmula petición de disculpas para una solicitud dirigida a una persona con mayor fuerza relativa, tanto si implica mayor grado de imposición como si no.

- Se observa que las fórmulas minimización de la imposición y agradecimiento son más comunes entre los hablantes colombianos.

- La mayoría de los casos de la fórmula expresión de la vergüenza fueron empleados por los colombianos. Es una de las diferencias más destacables entre los dos países según nuestros datos. Además esta fórmula suele ser utilizada especialmente para una petición a una persona con mayor fuerza relativa.

- En cuanto al uso de por favor o porfa, parecen ser utilizados más por los peruanos e, inesperadamente, es más común en los mensajes para amigos, es decir, personas con una menor fuerza relativa.

Como ya mencionamos, esta investigación es parte de un proyecto cuyo objetivo es describir las características 
Algunas estrategias para expresar cortesía en los escritos de petición en el español peruano y colombiano de las expresiones escritas de cortesía utilizadas por los hablantes de varios países hispanos, y posteriormente aplicar este conocimiento a la enseñanza de español como lengua extranjera. Por consiguiente, es imprescindible ampliar el área geográfica objetivo y llevar a cabo la misma investigación con hablantes de otros países. Asimismo, es posible que dentro de un mismo país existan diferencias según la edad, el género, entre otros factores, por lo que es necesario realizar dichas distinciones en el análisis de los datos. ${ }^{19}$

Este estudio tiene algunas limitaciones. En primer lugar, la relación entre el hablante y su jefe/jefa no fue especificada de manera concreta. Así, cada participante visualizó una dinámica de interacción individual, y asignó una mayor o menor distancia social a la relación con el interlocutor. Por otro lado, algunos participantes malinterpretaron una o más situaciones presentadas en la tarea y crearon mensajes que no pudieron ser considerados en el análisis. Y finalmente, existen numerosas expresiones empleadas para hacer una petición pero que no se han analizado detalladamente en este trabajo, como si fuera tan amable de, etc. Estos factores serán tomados en cuenta en futuras investigaciones.

19. Como docentes de español en Japón, una de nuestras metas es realizar un estudio con participantes japoneses estudiantes de español como lengua extranjera. El objetivo es contrastar sus datos con los de los hablantes nativos de español e identificar diferencias y similitudes. 
Algunas estrategias para expresar cortesía en los escritos de petición en el español peruano y colombiano

\section{Referencias bibliográficas}

1. Ballesteros Martín, F. J. (1999). La cortesía verbal. Análisis pragmático-lingüístico de las exhortaciones impositivas en inglés y en español: el ruego y el mandato (Tesis doctoral). Universidad Complutense de Madrid, Madrid.

2. Blum-Kulka, S., House, J. \& G. Kasper (1989). Cross-Cultural Pragmatics: Requests and Apologies. Norwood: Abex.

3. Bravo, D. (2003). Actividades de cortesía, imagen social y contextos socioculturales: una introducción. En D. Bravo (Ed.), Actas del Primer Coloquio del Programa EDICE: La perspectiva no etnocentrista de la cortesía: identidad sociocultural de las comunidades hispanohablantes (pp.98-108). Universidad de Estocolmo, Estocolmo. 4. Bravo, D. (1999). ¿Imagen positiva vs. Imagen negativa? Pragmática social y componentes del face. Oralia, 2, 155-184.

5. Bravo, D. (2008). Las implicaciones del estudio de la cortesía en contextos del español. Una discusión. Pragmatics, 18(4), 577-603.

6. Brown, P., \& Levinson, S. C. (1987). Politeness: Some Universals in Language. Cambridge: Cambridge University Press.

7. Cantamutto, L. y Vela Delfa, C. (2019). Estrategias pragmáticas en correos electrónicos académicos de Argentina y España. Lengua y Habla, 23, 202-223. https://www.researchgate.net/publication/337412771_ Estrategias_pragmaticas_en_correos_electronicos_academicos_de_Argentina_y_Espana_Pragmatic_strategies_ in_academic_e-mails_from_Argentina_and_Spain

8. César Vera, Y. (2014). Ciberatenuación en Venezuela: interacción entre profesores y estudiantes universitarios a través de correos electrónicos. Onomázein, 30, 1-17. http://onomazein.letras.uc.c1/04_NumeroDescarga/n30/ PantallaDescarga_30-1.html

9. Curcó, C. (1998). ¿No me harías un favorcito?: reflexiones en torno a la expresión de la cortesía verbal en el español de México y el español peninsular. En H. Haverkate et al. (Eds.), La pragmática lingüística del español - Recientes desarrollos (pp. 129-171). Amsterdam-Atlanta: Editions Rodpi.

10. Escandell Vidal, M. V. (2013). Introducción a la pragmática. Barcelona: Ariel.

11. Haverkate, H. (2003). El análisis de la cortesía comunicativa, categorización pragmalingüística de la cultura española. En D. Bravo (Ed.), Actas del Primer Coloquio del Programa EDICE: La perspectiva no etnocentrista de la cortesía: identidad sociocultural de las comunidades hispanohablantes (pp. 60-70). Universidad de Estocolmo, Estocolmo.

12. Jang, J. S. (2011). Cortesía sociocultural en las sociedades medellinense y japonesa: una introducción. Íkala, Revista de Lenguaje y Cultura, 16(28), 119-157.

13. Kawaguchi, M. (2016). Investigación preliminar sobre las expresiones de cortesía en los correos electrónicos 
Algunas estrategias para expresar cortesía en los escritos de petición en el español peruano y colombiano de los estudiantes japoneses de español como L2. Boletín de la Universidad de Estudios Extranjeros de Kobe, 66(2), 131-149 (Artículo en japonés).

14. Kawaguchi, M. (2018). Componentes culturales y pragmáticos en las clases de composición en Japón: el caso de los correos electrónicos de petición y de rechazo. M. Bargalló Escrivá et al. (Eds.) Léxico y cultura en LE/L2: corpus y diccionarios (pp. 343-352). Asociación para la Enseñanza del Español como Lengua Extranjera. https:// cvc.cervantes.es/ensenanza/biblioteca_ele/asele/pdf/28/28_0032.pdf

15. Montolío, E. (Coord.) (1999). Manual de escritura académica. Barcelona: Ariel.

16. Montolío, E. (Coord.) (2000). Manual práctico de escritura académica. Barcelona: Ariel.

17. Montolío, E. (Dir.) (2014). Manual de escritura académica y profesional. Barcelona: Ariel.

18. Noda, H. (2014). El sentido y la metodología para captar la diversidad de las expresiones de la cortesía. En H. Noda et al.(Eds.) La diversidad de las expresiones de la cortesía en japonés (pp. 3-20). Tokio: Editorial Kurosio. (Libro en japonés).

19. Real Academia Española: Diccionario de la lengua española, 23ª edición, [versión 23.4 en línea]. https://dle. rae.es

20. Shimizu, T. (2009). An Introduction to Interlanguage Pragmatics. Tokio: 3A Corporation. (Libro en japonés). 21. Trosborg, A. (1995). Interlanguage Pragmatics - Requests, Complaints and Apologies. Berlín: Mouton de Gruyter.

22. Yui, K. (2005). Gramática pedagógica del japonés para escribir. En H. Noda (Ed.) Gramática pedagógica del japonés para la comunicación (pp. 187-206). Tokio: Editorial Kurosio (Artículo en japonés). 